\title{
Pertinence des marqueurs microsatellites (SSR) et apport de la bioinformatique en vue de la valorisation de l'huile d'olive
}

\author{
Relevance of microsatellite markers (SSR) and bioinformatics \\ contribution for the valorisation of olive oil
}

\author{
Rayda Ben Ayed ${ }^{1}$ \\ ${ }^{1}$ Laboratoire des Procédés de Criblage Moléculaire et Cellulaire. Centre de Biotechnologie de Sfax, Université de Sfax, \\ Tunisie, raydabenayed@yahoo.fr
}

RÉSUMÉ. Le patrimoine génétique oléicole mondial est très riche en variétés d'olivier (Olea europaea L.), cultivées et spontanées, et l'huile qui en est extraite est d'autant plus diversifiée. Cette richesse est souvent ignorée ou méconnue dans les détails. Par ailleurs, tenant compte des exigences de plus en plus restrictives du marché de l'huile et des besoins du consommateur, les recherches sur l'amélioration et la sélection (variétale ou clonale) visent la valorisation du patrimoine variétal autochtone et son enrichissement à travers l'amélioration génétique. Habituellement, la caractérisation et l'identification des ressources génétiques de l'olivier ont été réalisés par des descripteurs morphologiques liés aux différentes composantes de l'arbre (fleurs, feuilles, fruits, ...). Cependant, ces caractères morphologiques se sont révélés insuffisants et obsolètes du fait de leur faible pouvoir de discrimination et de leur instabilité liée à l'interaction avec les facteurs environnementaux. Récemment, de nouvelles approches multidisciplinaires ont été proposées à l'aide de marqueurs moléculaires, en l'occurrence les marqueurs SSR, combinés à des analyses bioinformatiques.

ABSTRACT. The world olive genetic heritage is very rich in varieties of olive tree (Olea europaea L.), both cultivated and spontaneous, and more diversified is the oil extracted from them. However, this richness is often ignored or misunderstood in detail. Furthermore, taking into account the increasingly restrictive requirements of the oil market and the needs of the consumer, researches on improvement and selection (varietal or clonal) focus on the value of the indigenous varietal heritage and its enrichment through genetic improvement. Usually, the characterization and identification of the genetic resources of the olive tree have been carried out by morphological descriptors related to the different components of the tree (flowers, leaves, fruits, ...). However, these morphological traits have proved to be insufficient and obsolete due to their low discriminating power and their instability linked to the interaction with environmental factors. Recently, new multidisciplinary approaches have been proposed using molecular markers, namely SSR markers, combined with bioinformatic analyses.

MOTS-CLÉS. Ressources génétiques, Olivier, Marqueurs SSR, Bioinformatique.

KEYWORDS. Genetic resources, Olive tree, SSR markers, Bioinformatics.

\section{Introduction}

De nos jours, une attention particulière est accordée à la connaissance exacte de l'origine et de l'authenticité des produits agricoles afin d'obtenir la confiance du consommateur mais aussi de lui garantir la valeur ajoutée tant sur le plan qualitatif que sanitaire. L'huile d'olive est devenue un produit très recherché et sa demande provient de tous les continents ; particulièrement l'huile d'olive extra vierge, dont la qualité répond à des normes internationales strictes. La tendance actuelle se penche vers la production d'huiles labellisées telles que les AOC (Appellation d'Origine Contrôlée), IGP (Indication Géographique Protégée) ou DOP (Dénomination d'Origine Protégée) qui devraient garantir à la fois qualité et origine géographique du produit. Malheureusement, la composition variétale n'est généralement pas précisée sur l'étiquette du produit. Des huiles mono-variétales apparaissent mais leur origine est souvent vague (seule précision du pays d'origine) car elles ne satisfont qu'aux normes courantes (vierge extra, vierge, courante...). Pour la plus grande part du marché de l'huile d'olive, l'étiquetage des bouteilles est insuffisant pour connaître l'origine variétale, ni la provenance. De plus, de nombreuses dérogations aux règles du marché sont rapportées et parfois même des adjonctions 
d'huiles d'autres espèces, moins onéreuses, telles que l'huile de maïs ou de tournesol, ont été signalées en tant que fraude. La traçabilité des huiles d'olive a donc vu le jour et cela a fait l'objet de nombreuses recherches ces dernières années. Il s'agit d'une démarche qui consiste à donner la possibilité de retrouver la trace des différentes étapes et lieux de vie d'un produit, depuis sa création jusqu'à sa consommation et destruction. Plusieurs techniques reposant sur l'analyse de la composition chimique de l'huile d'olive (acides gras, polyphénols, antioxydants...), comme la chromatographie en phase gazeuse ou liquide ..., ont été appliquées et introduites dans le secteur oléicole pour étudier l'authenticité et l'origine variétales des huiles [1,2]. Cependant, plusieurs difficultés ont été rencontrées pour distinguer les variétés d'olivier, car leurs caractéristiques physico-chimiques sont fortement influencées par les conditions environnementales. Ainsi, cette traçabilité analytique de l'huile d'olive s'est révélée insuffisante du fait de son instabilité liée à l'interaction avec les facteurs environnementaux. De nouvelles approches complémentaires ont été proposées à l'aide de marqueurs moléculaires neutres basés sur le polymorphisme génétique. En effet, le progrès technologique associé à la biologie moléculaire a permis de développer des outils fiables et performants notamment les marqueurs moléculaires basés sur le polymorphisme de l'ADN nucléaire. Or, pour étudier la traçabilité de l'huile d'olive, il est important d'étudier en premier lieu la ressource génétique de l'olivier pour comprendre le patrimoine génétique afin de caractériser et évaluer les ressources génétiques de l'olivier et de résoudre des problèmes de confusion dans la gestion des variétés d'olivier. L'objectif de cet article est de passer en revue les différentes approches utilisées pour l'identification variétale de l'olivier d'une part, et d'autre part, d'illustrer l'importance de combiner les marqueurs moléculaires microsatellites et les outils bioinformatiques pour l'étude de l'authenticité de l'huile d'olive produite.

\section{Etude des ressources génétiques de l'olivier par les marqueurs moléculaires}

Les marqueurs moléculaires basés sur le polymorphisme de l'ADN nucléaire ont montré une grande aptitude à décrire la variabilité génétique ainsi que sa répartition au sein des populations et des espèces du genre Olea. Différents marqueurs moléculaires ont été appliqués comme les RAPDs [3, 4, 5, 6, 7], les AFLPs [8, 9, 10], les RFLPs [11], les SCARs [12, 13], les ISSRs [5, 14, 15], les SSRs [16, 17, 18, $19,20,21]$ et les SNPs [22, 23, 24, 25, 26, 27]. Les marqueurs moléculaires RAPD, AFLP, SCAR, ISSR, SSR et SNP ont été largement utilisés avec succès chez l'olivier à des fins diverses (Tab. 1);

- Caractériser la variabilité génétique des variétés [28, 29],

- Étudier la phylogénie et retracer l'histoire évolutive de l'espèce [6, 11]

- Vérifier la paternité et la compatibilité [19,30],

- Etablir et dessiner des cartes génétiques [7,31],

- Assigner des individus aux groupes géographiquement définis [29],

- Déterminer l'authenticité et établir la traçabilité des huiles pour la protection commerciale et la détection de fraudes $[32,33,34,35]$. 


\begin{tabular}{|c|c|l|}
\hline $\begin{array}{c}\text { Marqueurs } \\
\text { génétiques }\end{array}$ & Utilisation & \\
\hline \multirow{3}{*}{ RAPD } & $\mathrm{D}$ & $3,6,7,36,37,39$ \\
\cline { 2 - 3 } & $\mathrm{DG}$ & $3,4,5,40$ \\
\cline { 2 - 3 } & $\mathrm{AH}$ & 14,41 \\
\hline \multirow{3}{*}{ AFLP } & $\mathrm{DG}$ & 9 \\
\cline { 2 - 3 } & $\mathrm{D}$ & 8,13 \\
\cline { 2 - 3 } & $\mathrm{AH}$ & 42 \\
\hline \multirow{3}{*}{ SCAR } & $\mathrm{D}$ & 12 \\
\cline { 2 - 3 } & $\mathrm{AH}$ & 13 \\
\hline \multirow{3}{*}{ ISSR } & $\mathrm{DG}$ & 5 \\
\cline { 2 - 3 } & $\mathrm{AH}$ & 14 \\
\cline { 2 - 3 } & $\mathrm{P}$ & 15 \\
\hline \multirow{3}{*}{ SNP } & $\mathrm{DG}$ & 22,43 \\
\cline { 2 - 3 } & $\mathrm{DG}$ et D & $23,24,26,27$ \\
\cline { 2 - 3 } & $\mathrm{AGP}$ & $17,18,20,24$. \\
\hline \multirow{5}{*}{ SSR } & $\mathrm{DG}$ & $10,18,21,24,28,35,44,45$ \\
\cline { 2 - 3 } & $\mathrm{CG}$ & 31 \\
\cline { 2 - 3 } & $\mathrm{D}$ & $21,29,35$ \\
\cline { 2 - 3 } & $\mathrm{AP}$ & $12,17,19,20,30$ \\
\cline { 2 - 3 } & $\mathrm{AH}$ & $20,24,32,34$ \\
\hline
\end{tabular}

DG : diversité génétique; $\mathrm{AH}$ : analyse des huiles et traçabilité ; $\mathrm{D}$ : discrimination et différenciation ; AP : analyse de paternité ; CG : Cartographie génétique ; P : Analyse phylogénétique ; AGP : association génotype/phénotype.

Tableau 1. Les marqueurs génétiques et leurs utilisations pour étudier les génotypes d'olivier et les échantillons d'huile d'olive.

\section{Etude des ressources génétiques de l'olivier par les marqueurs microsatellites (SSR) :}

\subsection{Principe des marqueurs microsatellites (SSR) :}

Les microsatellites ou séquences simples répétées (SSR) sont des morceaux d'ADN répétés en tandem formés de mono, di, tri et tétra-nucléotides. Les microsatellites les plus courants sont (A)n, (TC)n, (TAT)n et (GATA)n, les valeurs de n pouvant aller de quelques unités à plusieurs dizaines.

Les microsatellites répondent à la définition typique d'un bon marqueur puisqu'ils sont codominants, multialléliques, en nombre illimité, et remarquablement abondants dans les génomes des eucaryotes, transférables et très polymorphes [46]. En raison de leur niveau élevé de polymorphisme, ils constituent un outil très puissant dans la détermination de la parenté et l'évaluation de la variabilité intra et inter-population. Ils sont considérés comme l'un des systèmes de marqueur les plus connus et sont largement utilisés dans les études de diversité génétique des plantes, de déséquilibre de liaison, des cartes génétiques et les techniques de sélection assistée par marqueurs. La reproductibilité, le polymorphisme élevé et le pouvoir discriminant des marqueurs SSR ont été mis en évidence par plusieurs études. Ils permettent par conséquent de mieux apprécier la diversité intra et inter-population d'une part, et un typage moléculaire précis, rapide et sans aucune ambiguité d'autre part. Les SSRs présentent un haut degré de polymorphisme et une forte hétérozygotie qui fait d'eux les marqueurs de choix dans les études génétiques. Ils sont beaucoup plus polymorphes que les polymorphismes de fragments de restriction (RFLP) et les polymorphismes d'un seul nucléotide (SNP). C'est la technique de PCR qui est utilisée pour révéler le polymorphisme des microsatellites. Une paire d'amorces spécifiques des bordures droite et gauche d'un microsatellite est utilisée pour amplifier le même 
microsatellite chez différents individus. En effet, chaque microsatellite est bordé par des séquences uniques qui lui sont propres. Les fragments d'amplification sont ensuite révélés par électrophorèse (Fig. $1)$.

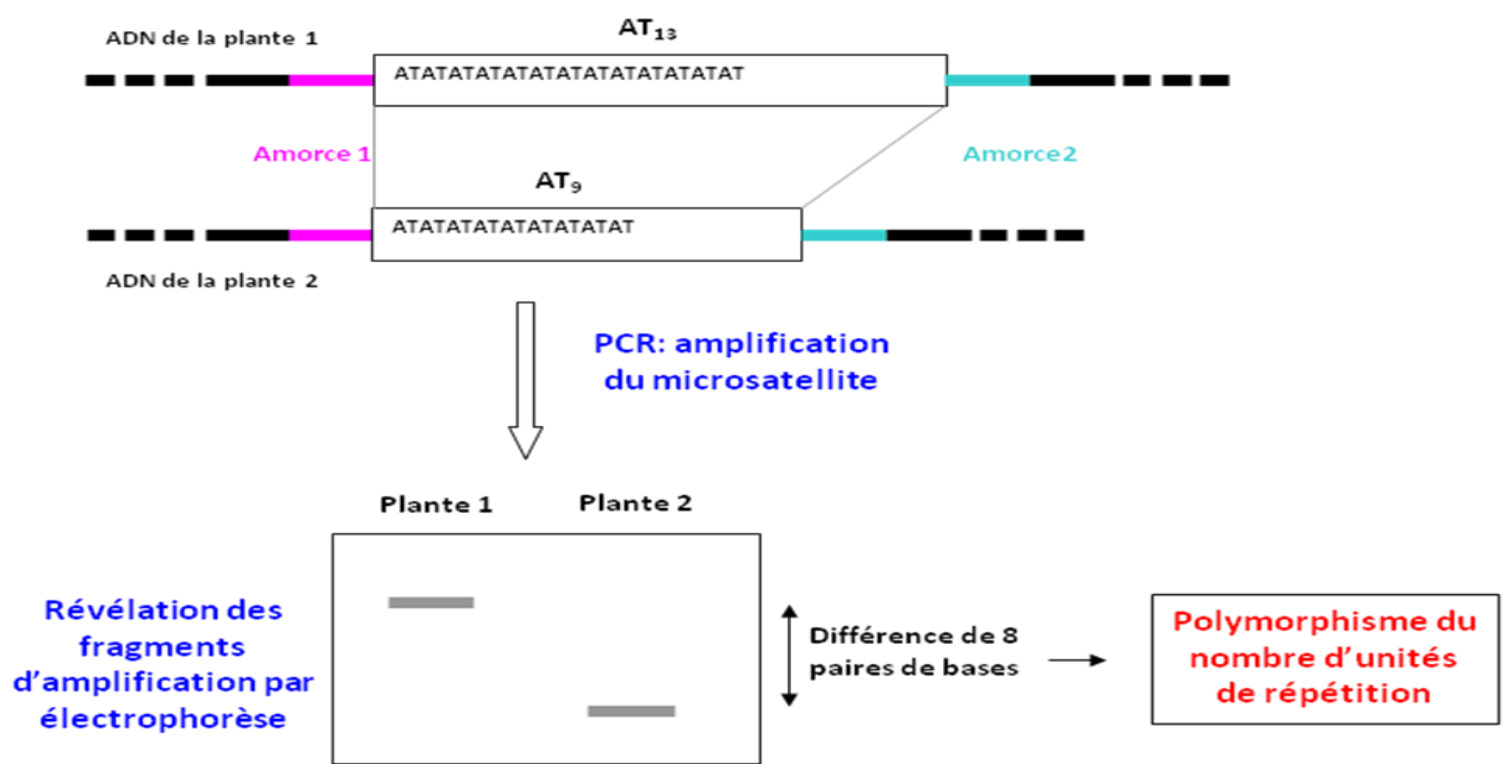

Marqueur = paire d'amorces spécifiques bordant le microsatellite

Figure 1. Illustration schématique du principe de la technique SSR.

\subsection{Application des marqueurs SSR}

Chez l'olivier, les marqueurs microsatellites ont été largement utilisés pour diverses études (Tab. 2). En effet, les SSRs ont montré un grand pouvoir de discrimination entre les variétés [10, 29]. Ces marqueurs sont considérés comme plus polymorphes que les marqueurs RAPD et AFLP et ils peuvent distinguer des variétés génétiquement très proches [3].

\begin{tabular}{|l|l|l|l|l|}
\hline $\begin{array}{l}\text { Nombre de } \\
\text { marqueurs } \\
\text { SSR }\end{array}$ & Origine & $\begin{array}{c}\text { Objectifs } \\
\text { de l'étude }\end{array}$ & Nombre de variétés & Références \\
\hline- & $\begin{array}{l}\text { Pays } \\
\text { méditerranéens }\end{array}$ & DG & 118 & 29 \\
\hline $\mathbf{1 1}$ & Tunisie & DG & 2 & 44 \\
\hline $\mathbf{9}$ & Tunisie & AH et D & 2 & 20 \\
\hline $\mathbf{8}$ & $\begin{array}{l}\text { Tunisie et des } \\
\text { variétés } \\
\text { introduites }\end{array}$ & $\begin{array}{l}\text { AH, DG et } \\
\text { D }\end{array}$ & 24 & 21 \\
\hline $\mathbf{9}$ & $\begin{array}{l}\text { Tunisie et des } \\
\text { variétés } \\
\text { introduites }\end{array}$ & AH, DG et & 22 & 35 \\
\hline $\mathbf{1 1}$ & Italie & DG & 211 & 45 \\
\hline $\mathbf{7}$ & Italie & AH & 7 & 47 \\
\hline $\mathbf{6}$ & Italie & AH & 1 & 32 \\
\hline $\mathbf{8}$ & $\begin{array}{l}\text { Italie, Portugal, } \\
\text { Espagne, France }\end{array}$ & D & 17 & 33 \\
\hline
\end{tabular}




\begin{tabular}{|l|l|l|l|l|}
\hline $\mathbf{7}$ & Italie & AH & 1 & 33 \\
\hline $\mathbf{1 2}$ & Croatie & DG & 27 & 48 \\
\hline $\mathbf{4}$ & Portugal & $\begin{array}{l}\text { DG, D et } \\
\text { AH }\end{array}$ & $\begin{array}{l}11 \text { cultivars et 12 } \\
\text { huiles d'olive } \\
\text { commerciales }\end{array}$ & 38 \\
\hline $\mathbf{7}$ & Espagne & D & 1 & 49 \\
\hline $\mathbf{6}$ & Italie & AH & 1 & 34 \\
\hline $\mathbf{1 6}$ & Italie & AH & 30 & 50 \\
\hline $\mathbf{1 1}$ & Espagne & AH & 21 & 51 \\
\hline $\mathbf{6}$ & Grèce & D & 26 & 52 \\
\hline
\end{tabular}

DG : diversité génétique ; $\mathrm{AH}$ : analyse des huiles ; $\mathrm{D}$ : discrimination et différenciation

Tableau 2. Utilisation des marqueurs microsatellites pour l'étude des génotypes d'olivier.

Plus de 150 marqueurs SSR ont été identifiés dans le génome de l'olivier. Une banque génomique d'olivier a été criblée par hybridation en utilisant comme sondes des motifs microsatellites [17] et les clones positifs ont été séquencés afin de désigner des amorces pour PCR. Il a ainsi été possible d'étudier le polymorphisme de 46 variétés et d'identifier 26 allèles SSR pour 5 loci différents. Carriero et al. [53] ont également identifié 10 marqueurs SSR dans le génome de l'olivier en utilisant la même méthode. Ils ont étudié la diversité génétique de 16 variétés d'Olea europaea. Ces marqueurs sont très utilisés pour construire des cartes de liaison dans le génome d'Olea [54] ainsi que pour retracer l'histoire et l'évolution de l'olivier [55]. Les microsatellites ont montré un fort potentiel pour résoudre les problèmes de synonymie, homonymie et fausse appellation entrainant beaucoup de confusions chez l'olivier [16, 18, 53, 54]. Les SSRs constituent d'excellents marqueurs génétiques appliqués dans l'étude de la diversité intra-variétale [18, 28, 54], la cartographie [31] et pour la caractérisation des ressources génétiques de l'olivier [3, 10, 33, 48, 49]. Sarri et al. [29] ont confirmé la puissance des marqueurs SSR pour l'identification de 118 cultivars provenant de différents pays méditerranéens. Tamaalli et al. [44] ont étudié la diversité des deux principales variétés tunisiennes (Chemlali et Chetoui) en utilisant 11 marqueurs SSR. Egalement, Ben Ayed et al. [20] ont étudié l'authenticité et la qualité des huiles des deux variétés tunisiennes majeures (Chetoui et Chemlali) en analysant les ADN isolés des huiles produites par ces deux variétés. Muzzalupo et al. [45] ont pu caractériser 211 variétés italiennes d'olivier à l'aide de 11 microsatellites et ont établi des corrélations entre l'origine géographique et les variétés d'olivier. Les microsatellites sont également des marqueurs très utilisés pour l'étude de paternité [17, 20, 21, 54]. Martins-Lopes et al. [38] ont examiné 11 échantillons d'huile d'olive portugais et 12 commerciaux (combinaison de marqueurs RAPD, ISSR et microsatellites) pour permettre la différenciation des variétés d'olives dans les huiles. Les microsatellites sont devenus des marqueurs moléculaires disponibles et fiables pour répondre aux questions de traçabilité et définir l'origine d'huile d'olive et détecter la présence des variétés indésirables et les manœuvres frauduleuses [32, 33, 34]. La plupart de ces publications avaient aussi pour objectif l'optimisation de l'extraction d'ADN de bonne qualité à partir de l'huile d'olive et d'identifier les marqueurs SSRs les plus intéressants dans la discrimination des variétés. En effet, Pasqualone et al. [47] ont utilisé 7 microsatellites (3DCA, 3GAPU et 1UDO) pour analyser l'huile extraite à partir de 7 variétés italiennes et étudier leur diversité génétique. Testolin et Lain [32] ont utilisé des ADN obtenus à partir de 3 échantillons d'huile d'olive vierge mono-variétale pour amplifier 6 loci microsatellites de la série UDO avec des amorces originales et internes, de manière à augmenter la sensibilité de la procédure.

\subsection{Marqueurs SSR et production d'huile d'olive}

La production d'huile d'olive ainsi que le rendement dépendent de facteurs environnementaux mais aussi génétiques. Pour des génotypes d'olive bien déterminés, il est possible de prévoir ou du moins estimer dans des conditions de culture optimales, la production et le rendement en huile d'olive. Les 
technologies reposant sur l'ADN peuvent jouer un rôle déterminant concernant la prédiction du rendement et de la qualité de l'huile d'olive, l'authentification et la traçabilité de l'huile d'olive et par conséquent la détection de fraude et le coupage des huiles. En général, les cultivars sont prédéfinis par certains caractères quantitatifs et qualitatifs tels que les niveaux de rendement en huile d'olive et sa qualité organoleptique. Les marqueurs SSR peuvent de ce fait constituer des indicateurs précoces de ces paramètres, et permettent par conséquent au producteur de connaitre et d'estimer la valeur de son huile d'olive. L'analyse de l'huile de l'olive via les marqueurs moléculaires se trouve confrontée à deux principaux obstacles techniques, l'extraction de l'ADN à partir d'une matrice liquide lipidique, et surtout la sélection de marqueurs moléculaires appropriés pouvant aboutir à des résultats significatifs $[20,56]$. En effet, le processus de filtration de l'huile d'olive, auquel s'ajoute l'action des nucléases, déprécie fortement la concentration de l'ADN dans l'huile d'olive [41]. Dès lors, plusieurs chercheurs ont tenté d'optimiser, avec plus ou moins de réussite, l'extraction d'ADN à partir de l'huile d'olive [57,8, 41]. En 2009, Ben Ayed et al. [20] ont réussi à définir un protocole d'extraction d'ADN à partir de la matrice huile d'olive avec des rendements fiables et suffisants pour les analyses ultérieures de type PCR, séquençage, clonage... L'ADN présent en quantités traces au niveau des échantillons d'huile d'olive a pu être ainsi extrait puis décodé, permettant-et permis ainsi de révéler son origine. L'utilisation des analyses basées sur la molécule d'ADN présente dans l'huile d'olive est une approche innovante et indépendante des conditions environnementales. Cette approche purement moléculaire permet, avec succès, de surmonter le problème de traçabilité des huiles monovariétales et multivariétales et vient compléter l'apport des caractéristiques biochimiques et organoleptiques pour la caractérisation et la traçabilité des huiles d'olive. Les résultats obtenus, vont permettre de dispenser une carte d'identité, une empreinte moléculaire, au produit avec exactitude, rapidité et à moindre coût. Cela va permettre d'accorder et d'ajouter une plus-value économique à ces huiles d'olive via l'application de cette stratégie de labellisation destinée à leur authentification. [21, 35].

Suite à l'initiation du projet de séquençage complet du génome de l'Olivier l'olivier [58], la recherche de marqueurs moléculaires de type SSR deviendra plus accessible et fructueuse [59]. Ainsi, l'utilisation de marqueurs moléculaires de choix vont permettre l'identification, à partir de l'empreinte génétique du cultivar, de la composition des huiles d'olive monovariétales [18, 57, 47]. L'approche SSR constitue une alternative, peu influencée par les conditions environnementales et les procédés d'extraction, permettant de reconnaitre l'empreinte génétique et par conséquent de caractériser quantitativement et qualitativement l'huile d'olive en fonction de la variété. Pour l'agriculteur, il s'agit certes d'un moyen qui va lui permettre d'effectuer des prévisions de rendement et de qualité de son huile et donc des estimations de profit et revenus financiers.

\section{L'apport des outils bioinformatiques en vue de l'étude des ressources génétiques de l'olivier et de la valorisation de l'huile d'olive}

La méthode d'étude des ressources génétiques de l'olivier et la traçabilité des huiles ne peuvent être efficaces que via une approche multidisciplinaire regroupant plusieurs domaines: la biologie moléculaire, la génétique, la biotechnologie végétale, la bioinformatique et la biostatistique. La bioinformatique est un domaine dont l'objectif est d'utiliser des outils informatiques et des modèles statistiques afin de cartographier et interpréter les données biologiques. De nombreux pays du bassin méditerranéen disposent d'une grande diversité génétique concernant l'olivier. Pour étudier les liens entre des variétés et entre des groupes d'une population d'oliviers, plusieurs équipes de recherche ont utilisé la classification phylogénétique. Cependant, les méthodes de phylogénie présentent une grande variabilité et se basent sur des modèles d'évolution dont la nature même restreint leur emploi. Un problème récurrent est lié à celui du mauvais usage de ces outils. Leur bon usage nécessite donc de connaitre les bases théoriques de ces méthodes et de ces modèles afin de mieux cerner leurs limites d'utilisation et c'est pourquoi il existe une forte demande d'améliorer les approches bioinformatiques que nous souhaiterons utilisées dans ce domaine. En effet, la phylogénie est un domaine en évolution constante et c'est grâce à d'importants progrès au niveau algorithmique que l'utilisation des méthodes 
relativement complexes de type approche bayésienne s'est répandue, alors qu'il y a une quinzaine d'années leur application était strictement limitée à des données de petite taille. Plus récemment, Ben Ayed et al. [27] ont étudié la diversité génétique de la plupart des variétés d'olive de table cultivées dans le monde moyennant une approche bayésienne basée sur le critère de productivité. Au même titre que de nombreux pays du bassin méditerranéen, le patrimoine tunisien actuel est riche en variétés d'oliviers réparties dans l'ensemble des régions du nord au sud du pays. Néanmoins, actuellement, seules des variétés d'intérêt général sont cultivées sur des surfaces significatives (particulièrement Chetoui au nord et Chemlali au centre et au sud). Malheureusement, peu d'agriculteurs possèdent sur leur terre des variétés peu diffusées et bien adaptées à leur région. Pour mieux connaitre les variétés d'oliviers présentes sur le territoire tunisien d'une part et d'autre part afin de mieux gérer ce patrimoine génétique et d'éviter les erreurs de dénomination (synonymie $=$ deux mêmes variétés portant deux noms différents, et homonymie $=$ un même nom est attribué à deux variétés différentes), il est indispensable de maintenir et de conserver cette richesse génétique. La conservation des ressources génétiques de l'olivier peut être effectuée au niveau de collections ex-situ à travers les différents domaines expérimentaux des laboratoires de recherche. Cependant et afin d'enregistrer les variétés ainsi que leurs caractéristiques de façon organisée et hiérarchisée, il est nécessaire de disposer d'une base de données. En effet et avec l'émergence des outils informatiques avancés, la conservation des génotypes et des caractéristiques des différentes variétés d'olivier devient une nécessité, et dans ce contexte, Ben Ayed et al. [35] ont implémenté la première base de données de génotypes d'oliviers du monde entier nommée OGDD (Olive Genetic Diversity Database) et hébergé au niveau d'un site web (http://www.bioinfo-cbs.org/ogdd/). De nos jours, la modélisation et la simulation des phénomènes biologiques en génétique ont acquis une grande popularité. Dans le domaine agricole, la modélisation appliquée sur des plantes permet de simuler la croissance des cultures, la productivité, et les propriétés agronomiques et de fournir des réponses claires aux agriculteurs dans le choix des génotypes appropriés en tenant compte de l'effet des scénarios de variations climatiques, les conditions édaphiques et la méthode de plantation. En effet, les modèles de simulation de cultures associés à la physiologie des plantes, aux paramètres environnementaux et à la génétique moléculaire ont été appliqués de différentes manières pour soutenir la sélection végétale et l'amélioration des plantes, en particulier pour la conception d'idéotypes, c'est-à-dire des plantes modèles, pour des cultures et environnements de culture différents [60]. Plusieurs équipes de recherche ont identifié et caractérisé des marqueurs SNP en utilisant la méthodologie GWAS (Genome-Wide Association Studies) pour mieux comprendre la variation génomique affectant les phénomènes complexes chez plusieurs espèces végétales. Par ailleurs, Shi et al. [61] ont simulé un modèle basé sur un processus pour prédire l'interaction de stérilité induite par une température élevée et aider à comprendre les caractéristiques de floraison d'une culture de riz sous une fluctuation de température. De plus, Krishnan et al. [62] ont développé des modèles basés sur la croissance des cultures afin de mieux comprendre les interactions complexes entre différentes variables environnementales qui influent sur la croissance et le rendement des cultures. Pour l'espèce Olea europaea L., Ben Ayed et al. [25] ont identifié deux marqueurs SNP étroitement associés à la composition en acides gras de l'huile d'olive tunisienne et ont conçu des blocs haplotypes, éventuellement responsables des interactions SNP associées aux acides gras en utilisant une simulation génétique statistique. En outre, la modélisation par simulation informatique a été utilisée pour étudier les relations entre les variétés de plantes. Ainsi, Ben Ayed et al. [21, 26, 35, 63] ont combiné une approche de simulation génétique et des données de marqueurs moléculaires afin de fournir une description plus précise de la structure génétique de l'olivier et de simuler la corrélation entre la productivité et les paramètres morphologiques, biologiques et physico-chimiques des principales variétés d'olives de table cultivées dans le monde entier afin de sélectionner le génotype d'olive de table approprié.

\section{Conclusions}

Les ressources génétiques d'oliviers cultivés et sauvages peuvent servir de réservoir pour sélectionner les gènes d'intérêt agronomique nécessaires aux programmes d'amélioration : résistance 
aux maladies, à la sécheresse, à une mauvaise qualité des sols, ou encore, facteurs d'une croissance accélérée, d'un rendement supérieur en huile, une meilleure qualité nutritive et organoleptique des huiles d'olive extraites. En effet, afin de pouvoir exploiter efficacement et de manière raisonnée la diversité génétique des oliviers cultivés et de leurs apparentés sauvages, il est nécessaire d'en avoir au préalable une bonne connaissance et de maitriser les outils de biologie moléculaire (en l'occurrence les marqueurs SSR et SNP) combinés avec des approches bioinformatiques permettant le transfert de caractères d'intérêt agronomique.

\section{Références bibliographiques}

[1] Khlif, M. Trigui, A., (1990) Olive cultivars investigations. Preliminary results. Acta. Hortic. 286, 65-68.

[2] Stefanoudaki, E., Kotsifaki, F., Koutsaftakis, A. (1999) Classification of virgin olive oils of the two major cretan cultivars based on their fatty acids composition. J. Am. Oil Chem. Soc., 76, 623-626.

[3] Belaj, A., Satovic, Z., Ismaeli, H., Panajoti, D., Rallo, L., Trujillo, I. (2003b) RAPD genetic diversity of Albanian olive germplasm and its relationships with other Mediterranean countries. Euphytica, 130, 387-395.

[4] Mekuria, G., Collins, G., Sedgley, M. (2002) Genetic diversity within an isolated olive (Olea europaea L.) population in relation to feral spread. SciHortic 94, 91-105.

[5] Gemas, V.J.V., Almadanim, M.C., Tenreiro, R., Martins, A., Fevereiro, P. (2004) Genetic diversity in the Olive tree (Olea europaea L. subsp. europaea) cultivated in Portugal revealed by RAPD and ISSR markers. Genet. Resour. Crop Evol. 51, 501-511.

[6] Besnard, G., Baradat, P., Chevalier, D., Tagmonount, A., Berville, A. (2001b). Genetic differentiation in the olive complex (Olea europaea L.) revealed by RAPDs and RFLPs in the rRNA genes. Genet Resour Crop Evol, 48, 165182.

[7] Khadari, B., Breton, C., Moutier, N., Roger, P.J., Besnard, G., Bervillé, A., Dosba, F. (2003) The use of molecular markers for germplasm management in a French olive collection. Theor. Appl. Genet. 106, 521-529.

[8] Busconi, M., Foroni,_C., Corradi, M., Bongiorni, C., Cattapan, F., Fogher, C. (2003) DNA extraction from olive oil and its use in the identification of the production cultivar. Food. Chem. 83,127-134.

[9] Sensi, E., Vignani, R., Scali, M., Masi, E., Cresti, M. (2003) DNA fingerprinting and genetic relatedness among cultivated varieties of Olea europaeaL. estimated by AFLP analysis. Sci.Hortic., 97(3-4), 379-388.

[10] Montemurro, C., Simeone, R., Pasqualone, A., Ferrara, E., Blanco, A. (2005) Genetic relationships and cultivar identification among 112 olive accessions using AFLP and SSR markers. J. Hort. Sci.Biotechnol. 80, 105-110.

[11] Besnard, G., Garcia-Verdugo, C., De Casas, R.R., Treier, U.A., Galland, N. and Vargas, P. (2008) Polyploidy in the olive complex (Olea europaea): evidence from flow cytometry and nuclear microsatellite analyses. Ann. Bot. (Lond) 101(1), 25-30.

[12] Busconi, M., Sebastiani, L., Fogher, C. (2006) Development of SCAR markers for germplasm characterisation in olive tree (Olea europaea L.). Mol. Breed. 17, 59-68.

[13] Pafundo, S., Agrimonti, C., Maestri, E., Marmiroli, N. (2007) Applicability of SCAR markers to food genomics: olive oil traceability. J. Agric. Food Chem., 55, 6052-6059.

[14] Pasqualone, A., Caponio, F., Blanco, A. (2001) Inter-simple sequence repeat DNA markers for identification of drupes from different Olea europaea L. cultivars. Eur. Food Res. Technol., 213, 240-243.

[15] Vargas, P., Kadereit, J.W. (2001) Molecular fingerprinting evidence (ISSR, inter-simple sequence repeats) for a wild status of Olea europaea L. (Oleaceae) in the Eurosiberian North of the Iberian Peninsula. Flora, 196, 142-152.

[16] Sefc, K.M., Lopes, M.S., Mendonça, D., Rodrigues Dos Santos, M., Laimer Da Câmara Machado, M., Da Câmara Machado, A. (2000) Identification of microsatellite loci in olive (Olea europaea) and their characterization in Italian and Iberian olive trees. Mol. Ecol., 9, 1171-1173.

[17] Rallo, P., Dorado, G., Martin, A. (2000) Development of simple sequence repeats (SSRs) in olive tree (Olea europaea L.). Theor. Appl. Genet., 101, 984-989.

[18] Cipriani, G., Marrazzo, M.T., Marconi, R., Cimato, A., Testolin, R. (2002) Microsatellite markers isolated in olive (Olea europaea L.) are suitable for individual fingerprinting and reveal polymorphism within ancient cultivars. Theor. Appl. Genet., 104, 223-228. 
[19] Diaz, A., Martin, A., Rallo, P., Barranco, D., de la Rosa, R. (2006) Self-incompatibility of 'Arbequina' and 'Picual' olive assessed by SSR markers. J. Am. Soc. Hort. Sci., 131, 250-255.

[20] Ben Ayed, R., Grati, N., Moreau, F., Rebaï, A. (2009) Comparative study of microsatellite profiles of DNA from oil and leaves of two Tunisian olive cultivars. European Food Research and Technology, 229 (5), 757-762.

[21] Ben-Ayed, R., Grati, N., Sans-grout,C., Moreau, F., Rebai, A. (2012) Characterization and authenticity of virgin olive oil (Olea europaea L.) cultivars by microsatellite markers. European Food Research and Technology 234(2), 263-271.

[22] Reale, S., Doveri, S., Díaz, A., Angiolillo, A., Lucentini, L., Pilla, F., Martín, A., Donini, P., Lee, D. (2006) SNPbased markers for discriminating olive (Olea europaea L.) cultivars. Genome, 15, 1193-1209.

[23] Santos Macedo, E., Cardoso, H.G., Hernandez, A., Peixe, A.A., Polidoros, A., Ferreira, A., Cordeiro, A., ArnholdtSchmitt, B. (2009) Physiologic responses and gene diversity indicate olive alternative oxidase as a potential source for markers involved in efficient adventitious root induction. Physiol. Plant, 137, 532-552.

[24] Ben-Ayed, R. Kallel, I., Ben Hassen, H., Rebai, A. (2014) SNP marker analysis for validating the authenticity of Tunisian olive oil. Journal of Genetics, 93, e148-e154.

[25] Ben Ayed, R., Ennouri, K., Ben Amar F., Moreau, F., Ali Triki M., Rebai A. (2017a) Bayesian and Phylogenic Approaches for Studying Relationships among Table Olive Cultivars. Biochemical Genetics, 55(4), 300-313.

[26] Ben Ayed, R., Ennouri, K., Ben Hlima H., Smaoui S., Hanana M., Mzid, R., Ercişli, S., Rebai A. (2017b) Identification and characterization of Single Nucleotide Polymorphism markers in FADS2 gene associated with olive oil fatty acids composition. Lipids in Health and Disease, 2017, 16:138.

[27] Ben Ayed, R. Ennouri, K., Ercişli, S., Ben Hlima H., Hanana M., Smaoui S., Rebai A., Moreau, F. (2018) First study of correlation between oleic acid content and $S A D$ gene polymorphism in olive oil samples through statistical and Bayesian modeling analyses. Lipids in Health and Disease, 2018,17:74.

[28] Lopes, M.S., Mendoca, D., Sefc, K.M., Sabino Gil, F., Da Camara Machado, A. (2004) Genetic evidence of intracultivar variability within Iberian olive cultivars. HortScience 39, 1562-1565.

[29] Sarri, V., Baldoni, L., Porceddu, A., Cultrera, N.G.M., Contento, A., Frediani, M., Belaj, A., Trujillo, I., Cionini, P.G. (2006) Microsatellite markers are powerful tools for discriminating among olive cultivars and assigning them to geographically defined populations. Genome, 49, 1606-1615.

[30] Mookerjee, S., Guerin, J., Collins, G., Ford, C. \& Sedgley, M. (2005) Paternity analysis using microsatellite markers to identify pollen donors in an olive grove. Theor. Appl. Genet. 111, 1174-1182.

[31] Wu, S.B., Collins, G., Sedgley, M. (2004) A molecular linkage map of olive (Olea europaea) based on RAPD, microsatellite, and SCARS markers. Genome, 47, 26-35.

[32] Testolin, R., Lain, O. (2005) DNA extraction from olive oil and PCR amplification of microsatellite markers. J. Food Sci., 70, 108-112.

[33] Doveri, S., Gil, F.S., Dıaz, A., Reale, -S., Busconi, M., Da Câmara, M.A., Martın, A., Fogher, C., Donini, P. \& Lee, D. (2008) Standardization of a set of microsatellite markers for use in cultivar identification studies in olive (Olea europaea L.). Scientia Horticulturae, 116, 367-373.

[34] Muzzalupo, I., Pellegrino, M., Perri, E. (2007) Detection of DNA in virgin olive oils extracted from destined fruits. Eur. Food Res. Technol., 224, 469-475.

[35] Ben Ayed, R., Ben Hassen, H., Ennouri, K., Ben Marzoug, R., Rebai, A. (2016) OGDD (Olive Genetic Diversity Database): A microsatellite markers' genotypes database of worldwide olive trees for cultivar identification and olive oil traceability. Database: The Journal of Biological Databases and Curation 2016, (DOI: 10.1093/database/bav090).

[36] Cresti, M., Linskens, H.F., Mulchay, D.L., Bush, S., Di, Stilo, V., Xu, M.Y., Vignani, R., Cimato, A. (1996) Preliminary communication about the identification of DNA in leaves and in olive oil of Olea europaea. Adv. Hort. Sci, 10, 105-107.

[37] Martins-Lopes, P., Lima-Brito, J., Gomes, S., Meirinhos, J., Santos, L., Guedes-Pinto, H. (2007) RAPD and ISSR molecular markers in Olea europaea L.: Genetic variability and molecular cultivar identification. Genet. Resour. Crop Evol. 54:117-128.

[38] Martins-Lopes, P., Gomes, S., Santos, E., Guedes-Pinto, H. (2008) DNA Markers for Portuguese Olive Oil Fingerprinting. J. Agric. Food Chem. 56 (24), 11786-11791.

[39] Claros, M.G., Crespillo, R., Aguilar, M.L., Canovas, F.M. (2000) DNA fingerprinting and classification of geographically related genotypes of olive-tree (Olea europaea L.). Euphytica, 116, 131-142. 
[40] Roselli, G., Petruccelli, L., Polsinelli, L., Cavalieri, D. (2002) Variability in five Tuscan olive cultivars (Olea europaea L.). J. Genet. Breed., 56, 51-60.

[41] Muzzalupo, I., Perri, E. (2002) Recovery and characterization of DNA from virgin olive oil. Eur. Food Res. Technol., 214 (6), 528-31.

[42] Pafundo, S., Agrimonti, C., Marmiroli, N. (2005) Traceability of plant contribution in olive oil by amplified fragment length polymorphisms. J. Agric. Food Chem., 53, 6995-7002.

[43] Muleo, R., Colao, M.C., Miano, D., Cirilli, M., Intrieri, M.C., Baldoni, L., Rugini, E. (2009) Mutation scanning and genotyping by high-resolution DNA melting analysis in olive germplasm. Genome 52, 252-260.

[44] Taamalli, W., Geuna, F., Banfi, R., Bassi, D., Daoud, D., Zarrouk, M. (2007) Using microsatellite markers to characterise the main Tunisian olive cultivars Chemlali and Chetoui. J. Hort. Sci. Biotechnol., 82, 25-28.

[45] Muzzalupo, I., Stefanizzi, F., Perri, E. (2009) Evaluation of Olives Cultivated in Southern Italy by Simple Sequence Repeat Markers. HortScience 44(3), 582-588.

[46] Toth, Z. G. and Jurka, J. (2000) Microsatellites in different eukaryotic genome, survey and analysis, Genome Res., 10, 1967-1981.

[47] Pasqualone, A., Montemurro, C., Caponio, F., Blanco, A. (2004) Identification of virgin olive oil from different cultivars by analysis of DNA microsatellites. J. Agric. Food Chem., 52, 1068-1071.

[48] Poljuha, D., Sladonja, B., Šetić, E., Milotić, A., Bandelj, D., Jakše, J., Javornik, B. (2008) DNA fingerprinting of olive varieties in Istria (Croatia) by microsatellite markers. Sci.Hortic.115, 223-230.

[49] Pasqualone, A., Montemurro, C., Summo, C., Sabetta, W., Caponio, F., Blanco, A. (2007) Effectiveness of Microsatellite DNA Markers in Checking the Identity of Protected Designation of Origin Extra Virgin Olive Oil. $J$. Agric.Food Chem., 55(10), 3857-3862.

[50] Alba, V., Sabetta, W., Blanco, A., Pasqualone, A. \& Montemurro, C. (2009) Microsatellite markers to identify specific alleles in DNA extracted frommonovarietal virgin olive oils. Eur. Food Res. Techno., DOI 10.1007/s00217009-1062-8.

[51] Vietina, M., Agrimonti, C., Marmiroli, M., Bonas, U., Marmiroli, N. (2011) Applicability of SSR markers to the traceability of monovarietal olive oils. Journal of the Science of Food and Agriculture, 91, 1381-1391.

[52] Roubos K., Moustakas, M. and Aravanopoulos, F.A. (2010) Molecular identification of Greek olive (Olea europaea) cultivars based on microsatellite loci. Genetics and Molecular Research, 9 (3), 1865-1876.

[53] Carriero, F., Fontanazza, G., Cellini, F., Giorio, G. (2002) Identification of simple sequence repeats (SSRs) in olive (Olea europea L.). Theor. Appl. Genet., 104, 301-307.

[54] De La Rosa, R., James, C.M., Tobutt, K.R. (2004) Using microsatellites for paternity testing in olive progenies. HortScience, 39, 351-354.

[55] Belaj, A., Munõz-Diez, C., Baldoni, L., Porceddu, A., Barranco, D. \& Satovic, Z. (2007) Genetic diversity and population structure of wild olives from the North-western Mediterranean assessed by SSR markers. Ann. Bot., 100, $449-458$.

[56] Enferadi, S. T. and Rabiei, Z. (2013) Challenges for Genetic Identification of Olive Oil, The Mediterranean Genetic Code - Grapevine and Olive, IntechOpen, DOI: 10.5772/53546.

[57] Breton, C., Claux, D., Metton, I., Skorski, G., Berville, A. (2004) Comparative study of methods for DNA preparation from olive oil samples to identify cultivar SSR alleles in commercial oil samples: possible forensic applications. Journal of Agricultural and Food Chemistry 52(3), 531-537.

[58] Cruz, F., Julca, I., Gómez-Garrido, J., Loska, D., Marcet-Houben, M., Cano, E., …Gabaldón, T. (2016). Genome sequence of the olive tree, Olea europea. GigaScience, 5, 29. https://doi.org/10.1186/s13742- 016-0134-5

[59] Sebastiani, L., Busconi, M. (2017) Recent developments in olive (Olea europaea L.) genetics and genomics: applications in taxonomy, varietal identification, traceability and breeding. Plant Cell Rep 36, 1345-1360.

[60] Slafer, G. A. (2003) Genetic basis of yield as viewed from a crop physiologist's perspective. Ann. Appl. Biol., 142, $117-128$.

[61] Shi, W.,__Muthurajan, R., Rahman, H., Selvam, J., Peng, S., Zou, Y., et al. . (2013) Source-sink dynamics and proteomic reprogramming under elevated night temperature and their impact on rice yield and grain quality. New Phytol., 197, 825-837. 10.1111/nph.12088. 
[62] Krishnan, P., Swain, D.K., -Bhaskar, B.C., Nayak, S.K., Dash, R.N. (2007) Impact of elevated $\mathrm{CO}_{2}$ and temperature on rice yield and methods of adaptation as evaluated by crop simulation studies. Agriculture, Ecosystems and Environment, 122, 233-242.

[63] Ben-Ayed, R., Grati, N., Rebai, A. (2013) An Overview of Authentication of Olive Tree and Oil. Comprehensive Reviews in Food Science and Food Safety, 12, 218-227. 